\title{
Tuning the Pseudospin Polarization of Graphene by a Pseudomagnetic Field
}

\author{
Alexander Georgi, ${ }^{\dagger}$ Peter Nemes-Incze, ${ }^{\dagger}$ Ramon Carrillo-Bastos, $^{\ddagger}, \nabla$ Daiara Faria,,$\|$
}

Silvia Viola Kusminskiy, ${ }^{\#}$ Dawei Zhai, $\nabla$ Martin Schneider, ${ }^{\#}$ Dinesh Subramaniam, ${ }^{\dagger}$ Torge Mashoff, ${ }^{\mathbb{I I}}$ Nils M. Freitag, ${ }^{\dagger \odot}$ Marcus Liebmann, ${ }^{\dagger}$ Marco Pratzer, ${ }^{\dagger}$ Ludger Wirtz, ${ }^{\perp}$ Colin R. Woods, ${ }^{\natural}$

Roman V. Gorbachev, ${ }^{\square}$ Yang Cao, ${ }^{\square}$ Kostya S. Novoselov, ${ }^{\square}$ Nancy Sandler, $\nabla$ and Markus Morgenstern $*^{\dagger}$

\begin{abstract}
${ }^{\dagger}$ II. Institute of Physics B and JARA-FIT, RWTH Aachen University, 52062 Aachen, Germany
${ }^{\ddagger}$ Facultad de Ciencias, Universidad Autónoma de Baja California, 21100 Mexicali, Baja California México

\#Dahlem Center for Complex Quantum Systems and Institut für Theoretische, Freie Universität Berlin, 14195 Berlin, Germany

IIJohannes Gutenberg-Universität, 55122 Mainz, Germany

•Instituto Politécnico, Universidade do Estado de Rio de Janeiro, 28625-570 Nova Friburgo, Brasil

${ }^{\S}$ Institute for Theoretical Physics II, University of Erlangen-Nüremberg, 91058 Erlangen, Germany

${ }^{\perp}$ Physics and Materials Science Research Unit, University of Luxembourg, L-1511 Luxembourg, Luxembourg

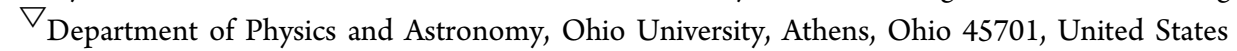

"Instituto de Física, Universidade Federal Fluminense, Niterói, 24210-340 Rio de Janeiro Brazil

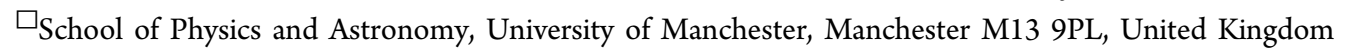

\section{Supporting Information}

\begin{abstract}
One of the intriguing characteristics of honeycomb lattices is the appearance of a pseudomagnetic field as a result of mechanical deformation. In the case of graphene, the Landau quantization resulting from this pseudomagnetic field has been measured using scanning tunneling microscopy. Here we show that a signature of the pseudomagnetic field is a local sublattice symmetry breaking observable as a redistribution of the local density of states. This can be interpreted as a polarization of graphene's pseudospin due to a strain induced pseudomagnetic field, in analogy to the alignment of a real spin in a magnetic field. We reveal this sublattice symmetry breaking by tunably straining graphene using the tip of a scanning tunneling microscope. The tip locally lifts the graphene membrane from a $\mathrm{SiO}_{2}$ support, as visible by an increased slope of the $I(z)$

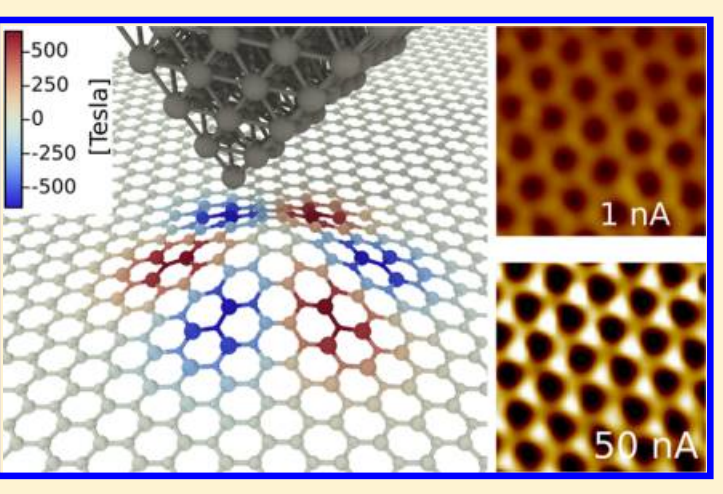
curves. The amount of lifting is consistent with molecular dynamics calculations, which reveal a deformed graphene area under the tip in the shape of a Gaussian. The pseudomagnetic field induced by the deformation becomes visible as a sublattice symmetry breaking which scales with the lifting height of the strained deformation and therefore with the pseudomagnetic field strength. Its magnitude is quantitatively reproduced by analytic and tight-binding models, revealing fields of $1000 \mathrm{~T}$. These results might be the starting point for an effective $\mathrm{THz}$ valley filter, as a basic element of valleytronics.
\end{abstract}

KEYWORDS: Graphene, strain, STM, pseudomagnetic field, pseudospin polarization, valley filter

train engineering in graphene has been pursued intensely $\checkmark$ to modify its electronic properties, ${ }^{1-4}$ mostly with a focus on deformations able to reproduce Landau level-like gapped spectra. $^{5-8}$ In addition to these effects, several theoretical works predict broken sublattice symmetry, measurable by the local density of states (LDOS) distribution in the presence of nonuniform strain. $^{9-16}$ This local sublattice symmetry breaking (SSB) implies a valley filtering property in reciprocal space that may be exploited for valleytronic applications via a clever and controlled tuning of strain patterns. ${ }^{17-23}$

In the Dirac description, the sublattice degree of freedom is represented by a pseudospin, and a sublattice symmetry

Received: November 22, 2016

Revised: February 13, 2017

Published: February 17, 2017 


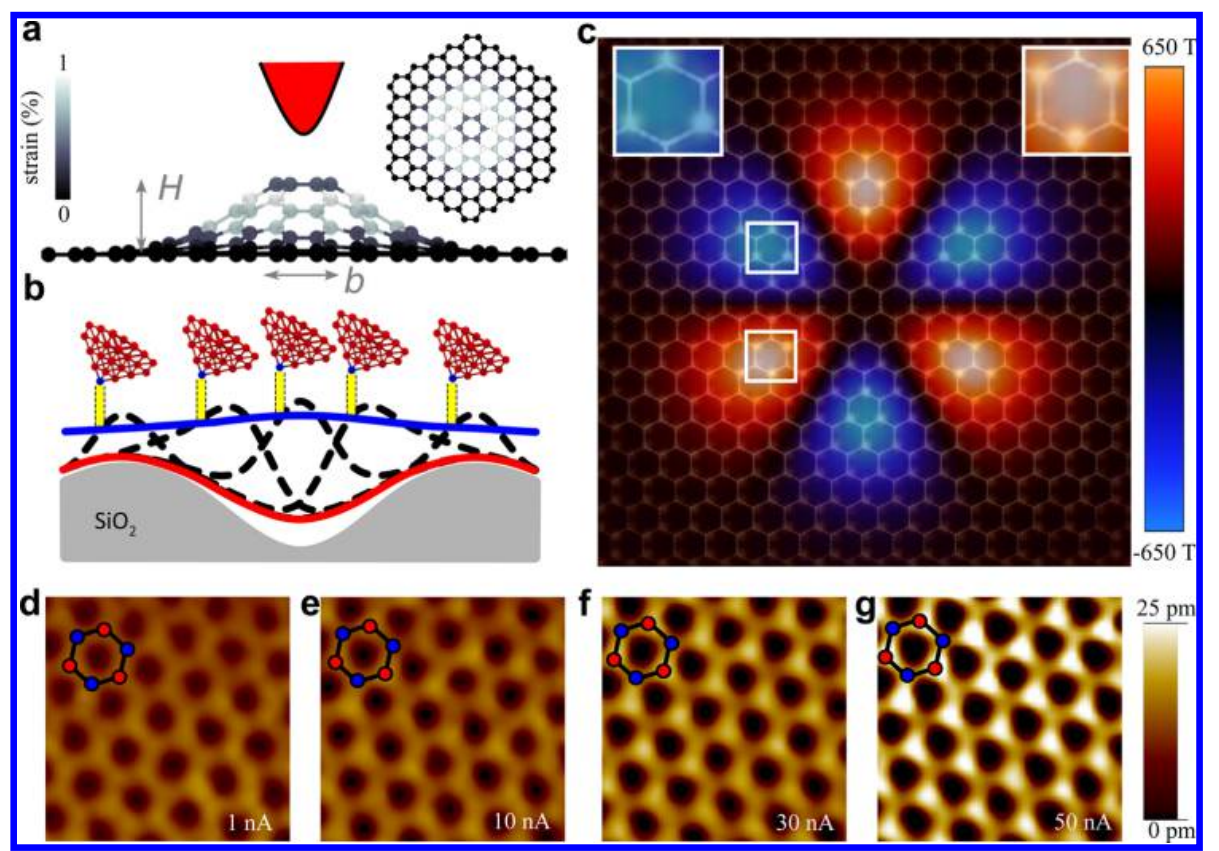

Figure 1. Sublattice symmetry breaking (SSB): (a) Gaussian deformation induced in graphene by the attractive van der Waals force of the STM tip (amplitude $H=1 \AA$, width $b=5 \AA$ ) as observed in molecular dynamics calculation of graphene on $\mathrm{SiO}_{2}$ (Figure $3 \mathrm{a}-\mathrm{c}$ ). Color code represents the induced strain. (b) The deformation (black dashed line) follows the scanning STM tip (red balls), leading to the apparent STM image (blue) lifted with respect to the relaxed one (red line). Yellow bar represents the tunneling current. (c) Color code: pseudomagnetic field pattern of the Gaussian deformation of panel a. ${ }^{15}$ The honeycomb lattice is overlaid with the LDOS magnitude of individual atoms, as calculated in nearest neighbor tightbinding ${ }^{13}$ marked by the brightness of the corresponding dots. White squares show areas of maximum $B_{\mathrm{ps}}$ magnified as insets. $(\mathrm{d}-\mathrm{g})$ Constant current STM images of the same graphene area on a $\mathrm{SiO}_{2}$ substrate recorded at varying currents as marked $\left(T=6 \mathrm{~K}, 1.3 \times 1.3 \mathrm{~nm}^{2}, V=0.5 \mathrm{~V}\right)$. A sketch of the graphene honeycomb lattice is overlaid with the different sublattices indicated by blue and red dots.

breaking is akin to a pseudospin polarization. It is thus tempting to assign the strain related SSB to an alignment of the pseudospin that occurs in the presence of a pseudomagnetic field. ${ }^{24}$ Below we present an intuitive understanding of the phenomenon, using the squared Dirac Hamiltonian and explain it qualitatively and quantitatively by a coupling of the pseudospin to the pseudomagnetic field that appears in the presence of strain. The interpretation is corroborated by experiments that use the tip of a scanning tunneling microscope (STM) to deliberately strain a graphene sample locally, in the form of a small Gaussian bump, and at the same time to map the imbalance of the local density of states (LDOS) at the sublattice level. Moreover, the measured sublattice contrast is quantitatively reproduced by an analytical model. ${ }^{15}$ These results provide a natural explanation for previous reports of SSB observed by STM in graphene, under nontunable mechanical deformations, ${ }^{7,8,25,26}$ which have so far not been attributed to a pseudospin polarization.

In the low-energy continuum Hamiltonian description for the electronic properties of graphene and other $2 \mathrm{D}$ materials with a honeycomb lattice, mechanical deformations lead to a vector potential $\vec{A}_{\mathrm{ps}}$, which is directly proportional to specific strain terms. ${ }^{5,27-30}$ The spatial dependence of $\vec{A}_{\mathrm{ps}}$ critically influences the dynamics of charge carriers. ${ }^{2}$ A mechanical deformation with $\nabla \times \vec{A}_{\mathrm{ps}} \neq 0$ results in an effective pseudomagnetic field, perpendicular to the graphene plane $B_{\mathrm{ps}}=\left(\nabla \times \vec{A}_{\mathrm{ps}}\right)_{z}$ that couples with different sign to states in the two valleys; ${ }^{27,29}$ i.e., it moves electrons in clockwise/ counterclockwise circles, respectively. An effective way to analyze the effect of $B_{\mathrm{ps}}$ on the pseudospin degree of freedom is realized by squaring the Dirac Hamiltonian. ${ }^{24,31}$ While the squared Hamiltonian describes the same physics as the original one, it also provides additional insight into the behavior of Dirac particles in a magnetic field. Following this procedure, for both valleys we obtain (Supplement S2-1):

$$
\begin{aligned}
& E^{2} \Psi^{\mathrm{A}}=v_{\mathrm{F}}^{2}\left[\vec{\pi}^{2}+e \hbar B_{\mathrm{ps}}\right] \Psi^{\mathrm{A}} \\
& E^{2} \Psi^{\mathrm{B}}=v_{\mathrm{F}}^{2}\left[\vec{\pi}^{2}-e \hbar B_{\mathrm{ps}}\right] \Psi^{\mathrm{B}}
\end{aligned}
$$

Here, $E$ is the energy, $\Psi^{\mathrm{A} / \mathrm{B}}$ is the wave function amplitude on the corresponding sublattice $\mathrm{A} / \mathrm{B}, \vec{\pi}=\left(\vec{p} \pm e \vec{A}_{\mathrm{ps}}\right)$ is the canonical momentum at each valley $( \pm), v_{\mathrm{F}}$ is the Fermi velocity, and $\vec{p}$ is the momentum measured from the respective Dirac points $\left(K, K^{\prime}\right)$. The first term $\left(\vec{\pi}^{2}\right)$ leads to Landau quantization, provided $B_{\mathrm{ps}}$ is homogeneous on the cyclotron radius scale, as observed by STM. ${ }^{6-8}$ The second term (with prefactor $v_{\mathrm{F}}^{2} e \hbar=658 \mathrm{meV}^{2} / \mathrm{T}$ ) corresponds to the coupling of $B_{\mathrm{ps}}$ to the graphene pseudospin. It appears with opposite signs at sublattices $A$ and $B$, shifting the energy of the respective states in opposite directions, thereby giving rise to a SSB, i.e., a pseudospin polarization. The SSB is identical for both valleys since the change in sign of $B_{\mathrm{ps}}$ between valleys compensates the sign change in sublattice space (see Supplement S2). An important feature of the $B_{\mathrm{ps}}$ pseudospin coupling is its locality, that allows to use the SSB as a local fingerprint for even strongly inhomogeneous $B_{\mathrm{ps}}$ (strain). The sublattice polarization resulting from the pseudomagnetic field has been predicted in several theoretical works. $^{9-16}$ The term that breaks the sublattice symmetry is sometimes referred to in the 


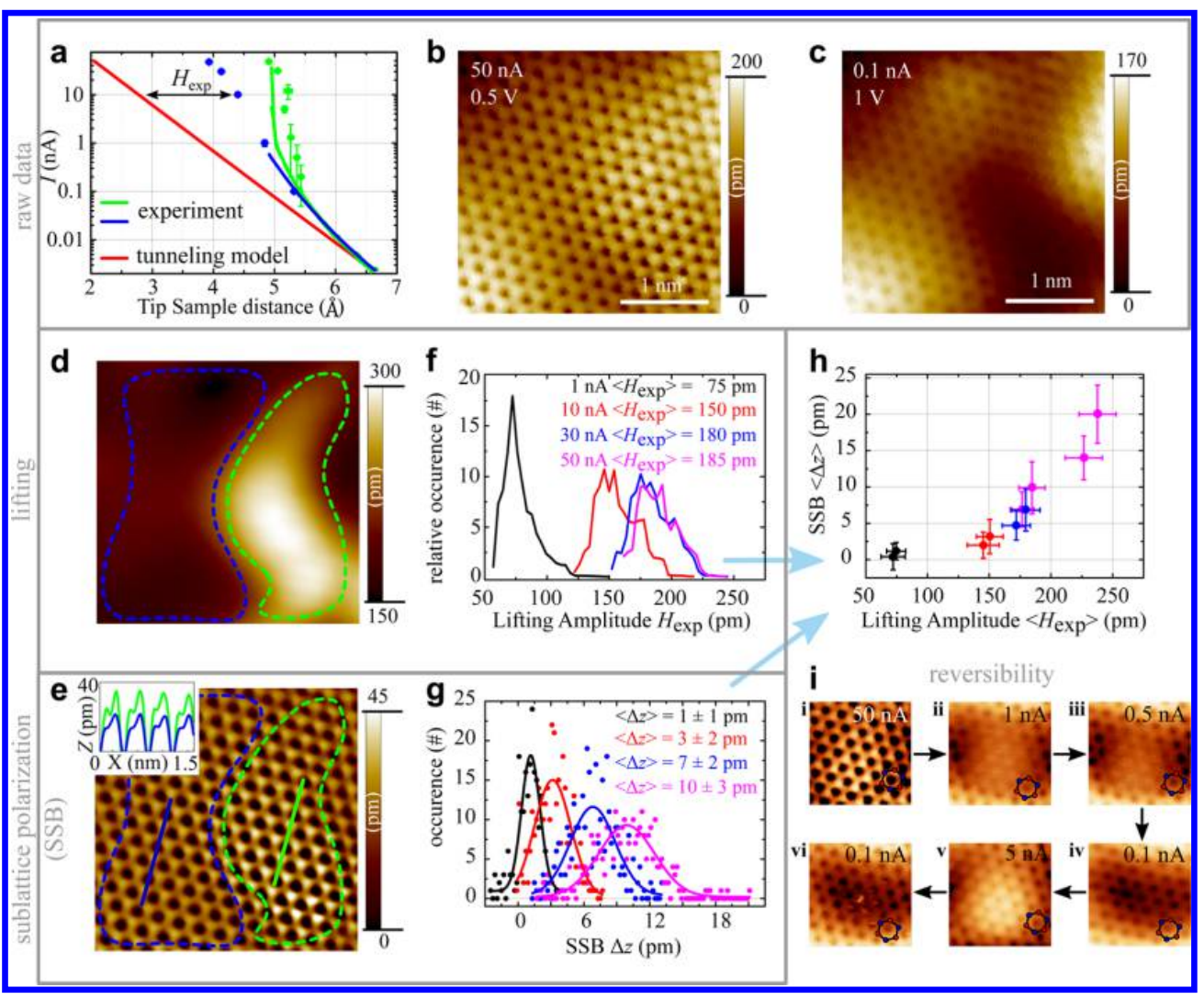

Figure 2. Relating lifting and SSB: (a) $I(Z)$ curves with logarithmic $I$ scale for regions with low/high lifting (see (d) blue/green areas). Dots (lines) are measured with (without) STM feedback loop (Supplement S5-2). Red line results from a 1D vacuum tunneling model ${ }^{41}$ using the work functions of graphene and tungsten. Black arrow indicates the deduced lifting amplitude $H_{\text {exp }}$ of graphene. (b, c) Raw STM data of the same area at different $I, V$, as marked. (d) Lifting amplitude $H_{\exp }$ at $I=50 \mathrm{nA}, V=0.5 \mathrm{~V}$ deduced from panels b and c (Supplement S5). (e) Atomic corrugation of panel $b$ obtained by subtracting the long-range morphology. Full lines mark the profile lines shown in the inset. Same curved, dashed lines in $d$ and $e$ (Supplement S5). (f) Histogram of $H_{\text {exp }}$ for the blue area in panel d, for different $I$ as marked, $V=0.5 \mathrm{~V}$. (g) Histogram of $\Delta z$ for the same area, at different currents $I$ (points) with Gaussian fits (lines). (h), Measured $\langle\Delta z\rangle$ with respect to $\left\langle H_{\text {exp }}\right\rangle$. Colors correspond to the accordingly colored tunneling current in panel f. Data from other sample areas than $(b, c)$ are included. (i) STM images of the same area $\left(V=0.5 \mathrm{~V}, 1.5 \times 1.5 \mathrm{~nm}^{2}\right)$ recorded consecutively as marked by the arrows at varying $I$.

literature as pseudo-Zeeman coupling. ${ }^{24,32,33}$ Its relation to the classic Zeeman effect for massive Fermions becomes obvious after squaring the Dirac Hamiltonian and developing it for the nonrelativistic limit $^{34}$ (see Supplement S2-1). Although for graphene the appropriate description is in terms of a massless Dirac equation, the analogy holds in the sense that the energy separation between the two pseudospin orientations is due to the coupling to the pseudomagnetic field. It is important to emphasize the difference between this term and another with the same expression, proposed as a gap opening perturbation for the Dirac Hamiltonian and unfortunately dubbed "pseudoZeeman" term, ${ }^{35}$ since a Zeeman coupling breaks a degeneracy without necessarily opening a gap at the Dirac point. As shown in Supplement Section S2, in order to open a gap the pseudomagnetic field should be even under inversion, while this is not the case for centrosymmetric deformations as the ones modeled in this work.

To produce $B_{\mathrm{ps}}$ and measure the resulting SSB, we use the tip of a scanning tunneling microscope which is known to locally strain graphene due to attractive van der Waals (vdW) forces. $^{36-38}$ Due to these forces, a Gaussian-shaped deformation forms below the $\mathrm{W}$ tip, locally lifting the graphene from its $\mathrm{SiO}_{2}$ substrate (Figure 1a), as evidenced by molecular dynamics calculations (see Supplement S3). The deformation moves along with the tip while scanning (Figure $1 b$, Supplementary Video). It has typical dimensions of $5 \AA$ halfwidth and $1 \AA$ height. The lifting height $H$ is tunable either by the tipgraphene distance $z$, adjusted by the tunneling current $I$, or by the locally varying adhesion forces of the substrate. ${ }^{36}$ The mechanical strain within the Gaussian deformation results in a 3 -fold symmetric $B_{\mathrm{ps}}$ pattern (color scale in Figure 1c), ${ }^{15}$ which shifts the local density of states (LDOS) in opposite directions at each sublattice. The resulting SSB, calculated by a nearest neighbor tight-binding model, ${ }^{13}$ maps $B_{\mathrm{ps}}$ in terms of sign and strength down to the atomic scale (Figure 1c) even while $B_{\mathrm{ps}}$ varies strongly on the scale of the pseudomagnetic length (0.4$1 \mathrm{~nm}$ ). A consequence of this strong variation is the lack of Landau levels in tunneling spectroscopy curves.

The key to measure the SSB is to tunnel into areas of large $B_{\mathrm{ps}}$, i.e., a few atomic distances offset from the deformation center. The inherent asymmetry of real STM tips makes this the common situation. We find SSB for $\sim 50 \%$ of the individually prepared tips; hence the tunneling atom is adequately offset with respect to the force center of the tip, i.e., the Gaussian maximum. Here, we present results from a single tip showing the strongest SSB within our experiments. However, comparable results are observed with other tips. In particular, if the tip remains unchanged, the same sublattice 

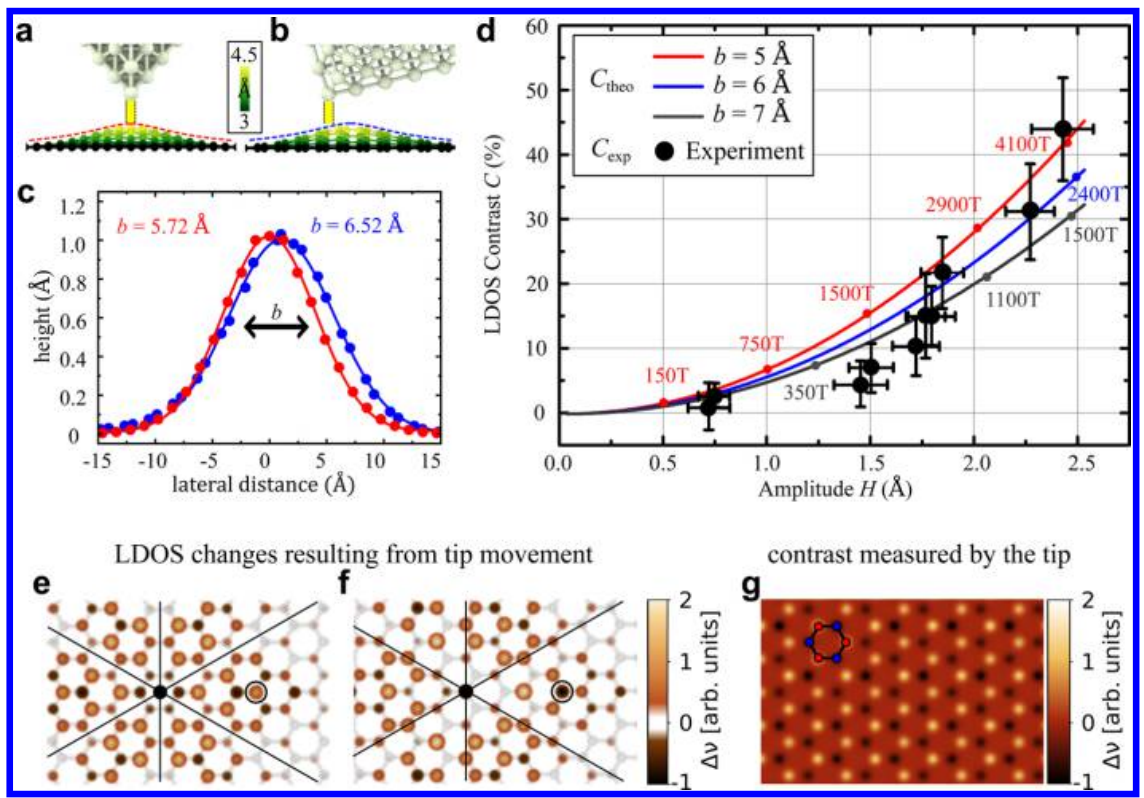

Figure 3. Comparing measured and calculated SSB: (a, b) Atomic configuration from molecular dynamics simulations of graphene on a $\mathrm{SiO}_{2}$ substrate in the presence of a pyramidal W (110) tip tilted by $\alpha=30^{\circ}$ with respect to the substrate normal, viewed from two perpendicular directions. The tunneling current is visualized as a yellow bar; the green color code marks graphene distance relative to the substrate, smallest tip sample distance: $z=3 \AA$. (c) Atomic positions, plotted as dots, along the dashed lines in a, b with Gaussian fits $h(r)=H \exp \left(-r^{2} / b^{2}\right)($ lines $)$. (d) Calculated and measured LDOS contrast $C$ as a function of the lifting amplitude $H$. $C_{\text {theo }}$ results from the continuum model (eq 3) and is plotted at $r$ $=b . C_{\text {exp }}$ results from the data of Figure $2 \mathrm{~h}$ using eq 4 . The corresponding maximum pseudomagnetic fields $B_{\mathrm{ps} \text {, max }}$ are marked at the dots on the full lines, in according color. All data are recorded with the same tip showing maximum SSB within our experiments. (e, f) LDOS patterns $(\Delta \nu=$ $\left.\nu_{\text {deformation }}-\nu_{\text {flat }}\right)$ resulting from TB calculation for a Gaussian deformation with $H=1 \AA, b=5 \AA$, and for different positions of the Gaussian center (black dot) with respect to the graphene lattice. Black ring corresponds to the tunneling position of the tilted tip being on sublattice A (e) and B (f). (g) Scanned LDOS pattern as observed by the tunneling atom at the black ring in e, f.

appears brighter in all areas of the sample (Supplement Figure S9), matching with the expectation that one always probes the same local region of the Gaussian, i.e. the same sign of $B_{\mathrm{ps}}$ (Supplementary Video, Figure 1b, c). STM images in Figure $1 \mathrm{~d}-\mathrm{g}$ demonstrate a controlled increase of SSB with increasing lifting force, i.e., increasing $I$ and decreasing $z$, respectively.

Next, we show that the sublattice contrast $C=2\left(\nu_{\mathrm{A}}-\nu_{\mathrm{B}}\right) /$ $\left(\nu_{\mathrm{A}}+\nu_{\mathrm{B}}\right)$, with $\nu_{\mathrm{A} / \mathrm{B}}$ the LDOS on sublattice $\mathrm{A} / \mathrm{B}$, can be related to $H$, the height of the Gaussian deformation, ${ }^{15}$ due to the dependence $B_{\mathrm{ps}} \propto H^{2}$. We estimate $H$ by comparing measured $\mathrm{I}(\mathrm{Z})$ curves $\left(\mathrm{Z}\right.$ : distance between tip apex and $\left.\mathrm{SiO}_{2}\right)$, with the standard exponential decay expected from the tunneling model. Therefore, we use the work functions of graphene $\Phi_{\mathrm{G}}=4.6 \mathrm{eV}^{39}$ and tungsten $\Phi_{\mathrm{W}}=5.3 \mathrm{eV}^{40}$ (red line in Figure 2a). The measured curves follow the usual dependence at large $Z$ but strongly deviate at smaller $Z$. Applying the tunneling model, ${ }^{41}$ the steepest areas (1-50 nA) would correspond to impossibly large work functions of $\Phi=$ $140 \mathrm{eV}$ (green) and $\Phi=21 \mathrm{eV}$ (blue). This indicates the local lifting of graphene toward the tip, which increases $I$ beyond the expected increase due to the tip movement. An elastic stretching of the tip is ruled out, since the same tips did not exhibit deviations from the tunneling model on $\mathrm{Au}(111)$. Furthermore, since the slope of the $\ln (I(Z))$ curves changes during the approach and varies across the sample surface, the previously reported ${ }^{42}$ slope change due to high momentum transfer during tunneling into the $\mathrm{K}$ points can also be ruled out. The lifting amplitude $H_{\exp }$ is thus well estimated as the difference between the measured $I(Z)$ and the tunneling model (red line) as marked. Variations of $H_{\exp }$ (green vs blue curve) indicate variations in the adhesion to the substrate. ${ }^{36,43}$
Importantly, a map of the observed lifting heights $H_{\exp }$ (Figure $2 \mathrm{~d}$ ) correlates with a map of the SSB (Figure 2e). SSB is consistently observed everywhere (Figure S9 of the supplement) and is reversibly tunable on the same area (Figure 2i).

In the following, we establish the relation between the apparent sublattice height difference $\Delta z$ and $H_{\text {exp }}$. We select areas of similar lifting height (for example, blue area in Figure $2 \mathrm{~d}$ ), subtract long-range corrugations, and determine $\Delta z$ for each pair of neighboring atoms (Figure 2e, Supplement S5). Resulting histograms of $H_{\exp }$ and $\Delta z$ with indicated mean values $\left\langle H_{\text {exp }}\right\rangle$ and $\langle\Delta z\rangle$ are shown for different $I$ in Figure 2f,g. The values of $\left\langle H_{\text {exp }}\right\rangle$ and $\langle\Delta z\rangle$ recorded on different areas and at different $z$, i.e., $I$, collapse to a single curve (Figure $2 \mathrm{~h}$ ). Areas with larger $\left\langle H_{\text {exp }}\right\rangle$ observed at the same $I$ are most likely caused by locally reduced adhesion to the $\mathrm{SiO}_{2},{ }^{36}$ while the observed lower liftings of $\left\langle H_{\exp }\right\rangle \approx 1.5 \AA$ are well reproduced by molecular dynamics calculations of graphene on flat, amorphous $\mathrm{SiO}_{2}$ with an asymmetric $\mathrm{W}$ tip in tunneling distance (see Supplement S3). Due to the much larger polarizability of W $\left(21.4 \AA^{3}\right)$ with respect to $\mathrm{Si}\left(6.81 \AA^{3}\right)$ and $\mathrm{O}\left(0.7 \AA^{3}\right)$, the graphene is lifted from the $\mathrm{SiO}_{2}$, even if the attractive dielectric forces between tip and graphene are neglected (Figure $3 \mathrm{a}-\mathrm{c}$ ). Importantly, the graphene below the tip is well-approximated by a Gaussian deformation. The observed LDOS sublattice contrast can thus be compared with the predicted analytic expression: ${ }^{15}$

$$
C_{\text {theo }}(r, \theta)=-\frac{2 \beta H^{2}}{b a} \sin (3 \theta) g(r / b)
$$


$\left(g(x)=\frac{1}{4 x^{3}}\left[1-e^{-2 x^{2}}\left(1+2 x^{2}+2 x^{4}\right)\right], \theta:\right.$ azimuthal angle, $r$ : distance from center, $\beta=3, a$ : lattice constant of graphene, and $b$ : width of the Gaussian deformation). To compare with our experimental results, we determine the LDOS contrast $C_{\text {exp }}$ from $\langle\Delta z\rangle$ (see Supplement S5) using

$$
\begin{aligned}
& C_{\exp }=2 \frac{\mathrm{e}^{\kappa\langle\Delta z\rangle}-1}{\mathrm{e}^{\kappa\langle\Delta z\rangle}+1} \\
& \text { with } \kappa=\sqrt{\frac{8 m_{\mathrm{e}}}{\hbar^{2}}\left(\frac{\Phi_{\mathrm{G}}+\Phi_{\mathrm{W}}}{2}-\frac{e|V|}{2}\right)}
\end{aligned}
$$

( $m_{\mathrm{e}}$ : free electron mass, $V$ : the sample voltage). The comparison is shown in Figure $3 \mathrm{~d}$ with $C_{\exp }$ values being consistent with $C_{\text {theo }}$ for deformation widths $b=5-7 \AA$, in excellent agreement with $b$ deduced from the molecular dynamics simulation (Figure $3 a-c$ ). Finally, we examine the effect of the deformation being moved with the tip across the graphene lattice (see Supplementary Video). Figure 3e,f displays the LDOS from a tight-binding (TB) calculation using two different central positions for the deformation, such that either sublattice A or B is imaged by the offset tunneling tip (black circle). The lateral shift of the tip preserves the sign of the SSB, while the observed contrast changes slightly to $6.5 \%$ (Figure 3g), from $6.9 \%$ in the static deformation. Conclusively, the model of pseudospin polarization describes our SSB data without any parameters which are not backed up by physical arguments. Note that we have carefully considered tip artifacts and several alternative explanations for SSB, all of which strongly fail either quantitatively or qualitatively to explain the experimental data (Supplement S4). Furthermore, strong SSB observed on a static graphene bubble (Supplement S7) further supports the straightforward pseudospin polarization scenario.

The observed pseudospin polarization dependent on $B_{\mathrm{ps}}$ adds an important ingredient to the analogy of graphene's Dirac charge carriers to ultrarelativistic particles. In turn, the changes in SSB might be used to probe $B_{\mathrm{ps}}$ on small length scales. ${ }^{44}$ Furthermore, the large values of $B_{\mathrm{ps}}(\sim 1000 \mathrm{~T})$ arising due to the dependence $B_{\mathrm{ps}} \propto \mathrm{H}^{2} / b^{3},{ }^{15}$ suggest the use of the strained region as a valley filter, ${ }^{21,22}$ operating on nanometer length scales and switchable with $\mathrm{THz}$ frequency (Supplement S8). Recently, valley currents with relaxation length of up to $1 \mu \mathrm{m}$ have been measured, ${ }^{45,46}$ but so far only in static configurations. Finally, the existence of strain induced SSB provides the first direct experimental evidence of the unique time reversal invariant nature of $B_{\mathrm{ps}}$. Being fundamentally different from a real magnetic field, this property could provide novel ground states dominated by many body interactions not achievable otherwise $^{47,48}$ or in combination with a real magnetic field of comparable magnitude could mimic the decoupling of a chiral flavor as observed in the weak interaction. ${ }^{49}$

\section{ASSOCIATED CONTENT}

\section{S Supporting Information}

The Supporting Information is available free of charge on the ACS Publications website at DOI: 10.1021/acs.nanolett.6b04870.

Supplementary sections S1-S8: relation between chiral symmetry, parity, and sublattice symmetry breaking in graphene; molecular dynamics calculation details; excluding alternative models for sublattice symmetry breaking; STM experimental details; tight binding calculation details; sublattice symmetry breaking in a graphene bubble; valley filter (PDF) Video (AVI)

\section{AUTHOR INFORMATION}

\section{Corresponding Author}

*E-mail: mmorgens@physik.rwth-aachen.de. Fax: +49-2418022306.

ORCID $\odot$

Peter Nemes-Incze: 0000-0002-1222-3020

Nils M. Freitag: 0000-0002-5043-6385

\section{Notes}

The authors declare no competing financial interest.

\section{ACKNOWLEDGMENTS}

We acknowledge discussions with M. I. Katsnelson, A. Bernevig, M. Krämer, W. Bernreuther, F. Libisch, C. Stampfer and C. Wiebusch, assistance at the STM measurements and sample preparation by C. Pauly, C. Saunus, S. Hattendorf, V. Geringer. We acknowledge financial support by the Graphene Flagship (Contract No. NECT-ICT-604391) and the German Research Foundation via Li 1050/2-2 (A.G., P.N.I., M.P., M.L. and M.M.); DFG SPP 1459 and the A. v H. Foundation (M.S., S.V.K.); CNPq No.150222/2014-9 (D.F.); NSF No. DMR1108285 (D.F., R.C-B., D.Z. and N.S.); PRODEP 2016 (R.C.B); FNR Luxembourg INTER/ANR/13/20/NANOTMD (L.W).

\section{REFERENCES}

(1) Amorim, B.; Cortijo, A.; de Juan, F.; Grushin, A.; Guinea, F.; Gutiérrez-Rubio, A.; Ochoa, H.; Parente, V.; Roldán, R.; San-Jose, P.; Schiefele, J.; Sturla, M.; Vozmediano, M. Phys. Rep. 2016, 617, 1-54.

(2) Castro Neto, A. H.; Pereira, V. M. Phys. Rev. Lett. 2009, 103, 046801 .

(3) Huang, M.; Pascal, T. A.; Kim, H.; Goddard, W. A.; Greer, J. R. Nano Lett. 2011, 11, 1241-1246.

(4) Zhang, Y.; Luo, C.; Li, W.; Pan, C. Nanoscale 2013, 5, 2616.

(5) Guinea, F.; Katsnelson, M. I.; Geim, A. K. Nat. Phys. 2010, 6, 3033.

(6) Levy, N.; Burke, S. A.; Meaker, K. L.; Panlasigui, M.; Zettl, A.; Guinea, F.; Neto, A. H. C.; Crommie, M. F. Science 2010, 329, 544547.

(7) Lu, J.; Neto, A. C.; Loh, K. P. Nat. Commun. 2012, 3, 823.

(8) Gomes, K. K.; Mar, W.; Ko, W.; Guinea, F.; Manoharan, H. C. Nature 2012, 483, 306-10.

(9) Wehling, T. O.; Balatsky, A. V.; Tsvelik, A. M.; Katsnelson, M. I.; Lichtenstein, A. I. EPL (Europhysics Lett. 2008, 84, 17003.

(10) Barraza-Lopez, S.; Pacheco Sanjuan, A. A.; Wang, Z.; Vanević, M. Solid State Commun. 2013, 166, 70-75.

(11) Moldovan, D.; Ramezani Masir, M.; Peeters, F. M. Phys. Rev. B: Condens. Matter Mater. Phys. 2013, 88, 035446.

(12) Neek-Amal, M.; Covaci, L.; Shakouri, K.; Peeters, F. M. Phys. Rev. B: Condens. Matter Mater. Phys. 2013, 88, 115428.

(13) Carrillo-Bastos, R.; Faria, D.; Latgé, A.; Mireles, F.; Sandler, N. Phys. Rev. B: Condens. Matter Mater. Phys. 2014, 90, 041411.

(14) Pacheco Sanjuan, A. A.; Wang, Z.; Imani, H. P.; Vanević, M.; Barraza-Lopez, S. Phys. Rev. B: Condens. Matter Mater. Phys. 2014, 89, 121403.

(15) Schneider, M.; Faria, D.; Viola Kusminskiy, S.; Sandler, N. Phys. Rev. B: Condens. Matter Mater. Phys. 2015, 91, 161407.

(16) Settnes, M.; Power, S. R.; Jauho, A.-P. Phys. Rev. B: Condens. Matter Mater. Phys. 2016, 93, 035456.

(17) Fujita, T.; Jalil, M. B. A.; Tan, S. G. Appl. Phys. Lett. 2010, 97, 043508.

(18) Low, T.; Guinea, F. Nano Lett. 2010, 10, 3551-3554. 
(19) Stegmann, T.; Szpak, N. New J. Phys. 2016, 18, 053016.

(20) Carrillo-Bastos, R.; León, C.; Faria, D.; Latgé, A.; Andrei, E. Y.; Sandler, N. Phys. Rev. B: Condens. Matter Mater. Phys. 2016, 94, 125422.

(21) Settnes, M.; Power, S. R.; Brandbyge, M.; Jauho, A.-P. Phys. Rev. Lett. 2016, 117, 276801.

(22) Milovanović, S. P.; Peeters, F. M. Appl. Phys. Lett. 2016, 109, 203108.

(23) Chaves, A.; Covaci, L.; Rakhimov, K. Y.; Farias, G. A.; Peeters, F. M. Phys. Rev. B: Condens. Matter Mater. Phys. 2010, 82, 205430.

(24) Sasaki, K.-I.; Saito, R. Prog. Theor. Phys. Suppl. 2008, 176, 253278.

(25) Xu, K.; Cao, P.; Heath, J. R. Nano Lett. 2009, 9, 4446-4451.

(26) Sun, G. F.; Jia, J. F.; Xue, Q. K.; Li, L. Nanotechnology 2009, 20, 355701.

(27) Kane, C. L.; Mele, E. J. Phys. Rev. Lett. 1997, 78, 1932.

(28) Suzuura, H.; Ando, T. Phys. Rev. B: Condens. Matter Mater. Phys. 2002, 65, 235412.

(29) Vozmediano, M.; Katsnelson, M.; Guinea, F. Phys. Rep. 2010, 496, 109-148.

(30) Cazalilla, M. A.; Ochoa, H.; Guinea, F. Phys. Rev. Lett. 2014, 113, 077201

(31) Aharonov, Y.; Casher, A. Phys. Rev. A: At., Mol., Opt. Phys. 1979, 19, 2461-2462.

(32) Katsnelson, M. I. Graphene: Carbon in Two Dimensions; Cambridge University Press, 2012.

(33) Kim, K.-J.; Blanter, Y. M.; Ahn, K.-H. Phys. Rev. B: Condens. Matter Mater. Phys. 2011, 84, 081401.

(34) Sakurai, J. J.; Napolitano, J. Modern Quantum Mechanics, 2nd ed.; Addison-Wesley, 2010.

(35) Mañes, J. L.; de Juan, F.; Sturla, M.; Vozmediano, M. A. H. Phys. Rev. B: Condens. Matter Mater. Phys. 2013, 88, 155405.

(36) Mashoff, T.; Pratzer, M.; Geringer, V.; Echtermeyer, T. J.; Lemme, M. C.; Liebmann, M.; Morgenstern, M. Nano Lett. 2010, 10, $461-465$.

(37) Klimov, N. N.; Jung, S.; Zhu, S.; Li, T.; Wright, C. A.; Solares, S. D.; Newell, D. B.; Zhitenev, N. B.; Stroscio, J. A. Science 2012, 336, 1557-1561.

(38) Wolloch, M.; Feldbauer, G.; Mohn, P.; Redinger, J.; Vernes, A. Phys. Rev. B: Condens. Matter Mater. Phys. 2015, 91, 195436.

(39) Yu, Y.-J.; Zhao, Y.; Ryu, S.; Brus, L. E.; Kim, K. S.; Kim, P. Nano Lett. 2009, 9, 3430-3434.

(40) Todd, C.; Rhodin, T. Surf. Sci. 1973, 36, 353-369.

(41) Tersoff, J.; Hamann, D. R. Phys. Rev. Lett. 1983, 50, 1998-2001.

(42) Zhang, Y.; Brar, V. W.; Wang, F.; Girit, C.; Yayon, Y.; Panlasigui, M.; Zettl, A.; Crommie, M. F. Nat. Phys. 2008, 4, 627-630.

(43) Geringer, V.; Liebmann, M.; Echtermeyer, T.; Runte, S.; Schmidt, M.; Rückamp, R.; Lemme, M.; Morgenstern, M. Phys. Rev. Lett. 2009, 102, 076102.

(44) Couto, N. J. G.; Costanzo, D.; Engels, S.; Ki, D.-K.; Watanabe, K.; Taniguchi, T.; Stampfer, C.; Guinea, F.; Morpurgo, A. F. Phys. Rev. $X$ 2014, 4, 041019.

(45) Gorbachev, R. V.; Song, J. C. W.; Yu, G. L.; Kretinin, A. V.; Withers, F.; Cao, Y.; Mishchenko, A.; Grigorieva, I. V.; Novoselov, K. S.; Levitov, L. S.; Geim, A. K. Science 2014, 346, 448-451.

(46) Shimazaki, Y.; Yamamoto, M.; Borzenets, I. V.; Watanabe, K.; Taniguchi, T.; Tarucha, S. Nat. Phys. 2015, 11, 1032-1036.

(47) Roy, B.; Assaad, F. F.; Herbut, I. F. Phys. Rev. X 2014, 4, 021042.

(48) Ghaemi, P.; Cayssol, J.; Sheng, D.; Vishwanath, A. Phys. Rev. Lett. 2012, 108, 266801.

(49) Sasaki, K.-I.; Saito, R.; Dresselhaus, M. S.; Wakabayashi, K.; Enoki, T. New J. Phys. 2010, 12, 103015. 